\title{
LONG-PERIOD VARIABLES AND PLANETARY NEBULAE
}

\author{
Donald H. MENZEL \\ (Harvard Observatory and Smithsonian Astrophysical Observatory, U.S.A.)
}

\begin{abstract}
A BSTRACT
The occasional appearance of a red giant or long-period variable in planetary nebulae poses a problem for theoretical astrophysics. Such a cool nuclear star would not ordinarily provide a source of ultraviolet radiation necessary for the excitation of the spectrum of a gaseous nebula.

One possible solution of this problem postulates the existence of intense magnetic fields in the star. Second, the star itself has a structure resembling that of a miniature, highly compressed planetary, with a high-temperature nuclear star at the centre and a distended atmospheric shell enveloping chiefly the stellar equator.

The magnetic field induces a sort of pumping action that creates the tire-shaped envelope from matter ejected near the poles. As this shell grows denser, it radiates like a stellar photosphere at low temperature. Eventually the shell becomes unstable and disperses outward to form and maintain the nebula. A quasi-periodic situation occurs, which explains the variation of light. Ultraviolet light absorbed during the minima, when the shell has vanished, adequately accounts for the nebular excitation. A wide variety of such symbiotic stars occurs, including repeating novae as well as the long-period variables.
\end{abstract}

Planetary nebulae, in general, owe their luminosity to the presence of high-temperature nuclear stars, whose intense ultraviolet radiation excites the gaseous nebulosity by fluorescence. The occasional appearance of a red giant or long-period variable, in planetary nebulae, seems completely anomalous. Paul Merrill referred to such associations as 'symbiosis', a biological term signifying an intimate association (often mutually beneficial) of two dissimilar organisms.

Such objects, observed near minimum, often show spectra characteristic of hot, blue stars - a fact that has led some astronomers, including myself, to suggest that these objects were actually double stars, a cool, red variable with an $\mathrm{O}$ or $\mathrm{W}$ companion.

More than 20 years ago, however, I suggested (Menzel, 1946) an alternative model, that giant $\mathrm{M}$ stars, in general, possess a relatively condensed and stable core, which by itself would be classed as spectral type $\mathrm{O}$ or W. A distended, variable, dynamic atmosphere surrounds this core. The atmosphere probably does not cover the entire star. Like an enormous doughnut or tire, it covers the equatorial belt, leaving the polar regions relatively clear, so that the hot core may freely radiate its ultraviolet energy into distant space.

In an accompanying paper (Menzel, 1968), I gave the magnetohydrostatic solution for the distribution of density and temperature in the gaseous envelope of a star.

Osterbrock and O'Dell (eds.), Planetary Nehulae, 386 389. "“ I.A.U. 
These formulae failed for planetary nebulae, but they seem adequate for a stellar envelope of relatively small radius.

The formulae are:

$$
\begin{aligned}
& p=p_{0}(r)+\frac{15 \mu^{2} a^{2} r^{2} \sin ^{2} \theta}{4 \pi\left(a^{2}+r^{2}\right)^{5}} \\
& \rho=\rho_{0}(r)+\frac{105 \mu^{2} a^{2} r^{2} \sin ^{2} \theta}{4 \pi G M\left(a^{2}+r^{2}\right)^{6}}
\end{aligned}
$$

where $p_{0}(r)$ and $\rho_{0}(r)$, functions of the radius $r$ only, define the pressure and density along the polar axis; also, $\mu$ is the magnetic moment. The parameter $a$ defines the radius of the currents producing the field. When $a=0$, the field becomes a simple dipole.

If we set these parameters equal to zero, we have a central star surrounded by an equatorial envelope, toroidal in shape.

The temperature distribution proves to be independent of the magnetic field. We have

$$
T=p m / k \rho=G M m\left(a^{2}+r^{2}\right) / 7 k r^{3},
$$

where $m$ is the molecular mass and $k$ Boltzmann's constant. For the Sun, with $a$ set equal to the radius, we find the temperature at the inner boundary,

$$
T_{a} \sim 4 \times 10^{6}
$$

a value suggestive of that for the inner corona. The number of atoms per $\mathrm{cm}^{3}$, from (2), at the equator for $r=a$, with

$$
\mu^{2}=2 H^{2} a^{6}
$$

where $H$ is the field intensity at the pole, becomes

$$
n=\rho / m=1.4 \times 10^{8} H^{2} \sim 3.5 \times 10^{9} / \mathrm{cm}^{3},
$$

a figure also very close to that of the base of the corona, with $H=5$ gauss.

I have not intended this demonstration to be a model of the solar corona; but the close agreement of the predicted temperature and density tend to confirm the validity of the model for its use in extended atmospheres. In summary, we may expect the temperature and density in a stellar envelope to follow laws like those of (3) and (2). However, if the atmospheres are very distended, we must adopt a value much larger than that of the stellar radius. The currents will be mainly in the distended atmosphere rather than in the central star. The steady state for which these distributions apply will almost certainly not exist.

A magnetohydrodynamic study of bipolar sunspots (Menzel and Shore, 1966) shows that solar flares probably result from a type of gaseous flow associated with strong magnetic fields. A search for magnetostatic or even steady-state solutions of 
the basic equations indicates that no such solutions exist for bipolar spots. In a steadystate solution, e.g., one seeks to find a gaseous flow along the magnetic lines of force. However, both spots of the pair attempt to 'pump' gas into the region between the spots. The pressure and temperature mount in this region until the magnetic field can no longer restrain the gas. The field expands, forming a shock wave that rushes over the solar surface.

Much the same thing can happen in a star containing a strong magnetic field. A static solution would require that regions near both magnetic poles be cooler and at lower pressure than the surroundings. Flow of heat into the regions, however, would prevent the static condition from ever occurring. Instead, each pole would begin to pump matter outwards, along magnetic lines of force, into the equatorial envelope of the star. The gradual accumulation of matter eventually leads to formation of a distended shell over the equator. As the opacity rises and radiative transfer takes control, the temperature falls. The shell converts the high-temperature radiation from the stellar nucleus into low-temperature radiation from the effective photosphere. The outer shell emits a spectrum characteristic of a long-period variable.

As for the sunspots, the pumping cannot continue indefinitely. Eventually, rising pressures cause the stellar magnetosphere to expand and suddenly - like an overinflated balloon - to break and discharge its contents into space. Thus, the expanding shells gradually coalesce to form the gaseous envelope of the planetary. And the ultraviolet radiation of the core, absorbed during minimum light, when the shell is nonexistent or incomplete, provides adequate energy for the nebula to shine steadily. The nebular gas has relaxation times far longer than the period of the associated variable.

Since the consecutive shells form and disperse over the equator, the ejecta over millenia conform at least roughly to the original toroid shape, distorted and expanded, but still toroidal. Viewed from the poles the nebula takes the form of a ring; from the equator it appears as two separate patches on either side of the star.

Fragments of the magnetic field may still persist in the expanding gas and serve to bind it together. Thus, many nebulae, such as the helical nebula in Aquarius, seem to consist of many small condensations rather than uniform continua. Each condensation could have resulted from a single outburst. (Could comets have arisen in some such manner?)

This model suggests that all planetary nebulae were once symbiotic objects. Conversely, it implies that all Mira-type and associated variables may eventually end up as standard planetaries. It further indicates a hitherto unsuspected kinship between giant $M$ 's, in general, long-period variables in particular, and the $\mathrm{W}-\mathrm{R}$ stars that so often appear as planetary nuclei. Do any of these stars exhibit light variation?

Symbiotic stars include a variety of types. The repeating nova, RS Ophiuchi, prominently displays the red coronal line of Fex at $\lambda 6374$ at a time when the associated star shows the $\mathrm{Ti} \mathrm{O}$ bands characteristic of an M-type spectrum. Such a line requires for its excitation temperatures of the order of a million degrees. $Z$ Andromedae, 
AX Persei, RX Puppis, T Coronae Borealis, RW Hydrae, CI Cygni, and AG Pegasi are other well-known examples of late-type stars having nebular characteristics. $\mathrm{R}$ Aquarii appears to be a typical long-period variable, except for its occurrence as the nuclear star of a planetary nebula. Sargent's (1966) recent discovery of forbidden lines of $\mathrm{N}_{\text {II }}$ in the spectrum of HR 8752, a G0 star, extends the relationship to stars other than M's.

\section{References}

Menzel, D.H. (1946) $\quad$ Physica, 12, 768.

Menzel, D.H. (1968) in the present volume, p. 279.

Menzel, D.H., Shore, B.W. (1966) in Comitato Nazionale per le Manifestazioni Celebrative del IV Centenario della Nascità di Galileo Galilei, Ed. by G. Barbera.

Sargent, W.L.W. (1966) Q. J. R. astr. Soc., 7, 222.

\section{DISCUSSION}

Kippenhahn: Several years ago Schwarzschild and Stothers determined the eigenperiods of models for the ascending branch of globular clusters. The periods they derived agreed quite well with the observed periods of Mira stars. We have also computed eigenperiods for red-giant models and got periods of the order of that of normal Mira variables. I therefore have the feeling that the Mira stars are really pulsating stars as the Cepheids are. In your model a torus-like figure is pulsating. Have you estimated eigenperiods and got similar agreement?

Feast: The mutilation of the hydrogen lines in Mira variables which is generally taken to imply a fairly dense overlying cool atmosphere is explained by your model. 